\title{
INTERFERING WITH NOMOLOGICAL NECESSITY
}

\section{Abstract}

\begin{abstract}
David Armstrong's law-maker, nomological necessity $(\mathrm{N})$, is a second order relational universal that holds between state of affairs types, e.g., $N(F, G)$. With only a small proviso, nomological necessity is supposed to instantiate as the causation of its second relatum, G, whenever its first relatum, F, instantiates. - In this paper, I will show that there is some friction within this theory when we consider that causal processes can be prevented and interfered with. The above mentioned proviso is supposed to handle these case but, so I argue, it fails to do so. - The critique here presented generalises to any theory of lawhood that utilises a kind of necessitation as lawmaker. Thus, Armstrong's case can serve as a sample for all such theories. Plausible means of resolving the difficulties will be presented.
\end{abstract}

Keywords: Armstrong, laws of nature, causation, nomological necessity, prevention and interference, exceptions, ceteris paribus

ca. 10,000 words

\section{Nomological NeCESSITY AND ITS RELATION TO CAUSATION}

Remember David Armstrong's theory of lawhood: a law of nature is identified with a second order relational universal, called "nomological necessity" (short: "N"), holding between universals. That is, Fs are $G$ s is a law iff $\mathrm{N}(\mathrm{F}, \mathrm{G}){ }^{1}$

What is nomological necessitation? Armstrong relates it closely to causation. Nomological necessity is, he claims, the relation-type of which singular causation is the token instantiation:

Singular causation is no more than the instantiation of this type of relation in particular cases. When we experience singular causation, what we are experiencing is nomicity, law-instantiation. (Armstrong 1997: 227)

Armstrong writes in later publications " $\mathrm{C}(\mathrm{F}, \mathrm{G})$ " or " $\left(\_1\right.$ being $\left.\mathrm{F}\right)$ causes (_2 being G)" (cf. Armstrong 1997: 230) rather than N(F, G) as a notation for the law-relation so that the close relation between nomological necessity and causation-the instantiation of necessity just is causation-is immediately obvious.

\footnotetext{
1 To do full justice to Armstrong's theory I should say that laws are nomic/nomological relations between state of affairs types with the property involved being a universal. A state of affairs type is a gutted state of affairs. It is what ' $a$ is $F$ ' becomes when we take away the object ' $a$ ' in abstraction: ' will nonetheless often speak of nomic necessity holding between universals for nothing hinges on that matter for the issue under concern here (cf. Armstrong 1997: 28-9).
} 
I aim to show in this paper that we have reasons to doubt that this direct identification can be made and that interferences and preventions pose unexpected metaphysical problems for Armstrong's theory. ${ }^{2}$

In fact, any theory of laws that is based on a form of necessity (metaphysical necessity included) will face these problems. I will first present these difficulties and then offer tentative suggestions for their solution.

\section{The InfERENCE Problem}

My starting point is to ask how we get from the law, $N(F, G)$, to the goings on in the world and especially the regularities we observe in nature. How does, if it does, N(F, G) entail $\forall \mathrm{x}(\mathrm{Fx} \supset \mathrm{Gx})$ ? Van Fraassen was the first to ask this question- "What information does the statement that one property necessitates another give us about what happens [...]?" (van Fraassen 1989: 96) - he called the related riddle the "inference problem", and thought it would not be solvable for Armstrong (cf. van Fraassen's, 1989: 96ff; and also Armstrong 1997: 228). ${ }^{3}$

Yet, Armstrong gave a convincing answer to the inference problem along the following lines: $F$ and $G$ are universals and so is $\mathrm{N}$. It holds as a second-order state of affairs between $F$ and $G: N(F, G)$. Now, if the universal $F$ is instantiated so must be $N$ simply because $\mathrm{N}(\mathrm{F}, \mathrm{G})$ holds. Again: if $\mathrm{N}(\mathrm{F}, \mathrm{G})$ holds $\mathrm{N}$ cannot but instantiate if $\mathrm{F}$ instantiates. $\mathrm{N}$ is dragged along by $\mathrm{F}$ (so to speak). The instantiation of $\mathrm{N}$, however, "is the causality instantiated in the situation". Hence, the universal G must be tokened as well since it is caused by the F-token qua N's instantiation. Accordingly, any instantiated F causes a $G$ and so, as a consequence, $\forall x$ (Fx $\supset$ Gx). Therefore, $N(F, G)$ does, indeed, ensure $\forall \mathrm{x}(\mathrm{Fx} \supset \mathrm{Gx})$ and "this would seem to solve the inference problem" (Armstrong 1997: 228).

\section{The IN-FERENCE Problem, Logically CONSTRUEd}

So far so good, but Armstrong denies that the entailment as sketched above goes through as easily as I made it seem: "The entailment actually holds only for the case where it is given that nothing further interferes." (Armstrong 1997: 230) And, for Armstrong, "it seems to be always possible that for any antecedent of a law there exists $[\ldots]$ an

\footnotetext{
2 One objection to Armstrong's theory goes like this: not all laws are causal laws-there are also the synchronic laws of co-existence like 'electrons have negative charge' or Pauli's exclusion principle, etc. How can, for those cases, N's instantiation be causation? Are electrons caused to be negatively charged? Although this is an interesting point in its own right (which Armstrong has acknowledged (cf., for example, (Armstrong 2004: 134)) it is not the subject of my paper. Rather, I aim to show that even for the causal laws themselves causation cannot be N's instantiation.
}

${ }^{3}$ Van Fraassen (van Fraassen 1989: 96ff) confronted Armstrong with two problems, one being the inference problem as above, the other being the identification problem: what exactly is nomological necessity? I have given the ontological part of Armstrong's answer to the latter question already: nomological necessity's tokeninstantiation is causation, i.e., nomological necessity is, so to speak, causation's universal. This idea has not always been part of Armstrong's theory. As a matter of chronological fact, Armstrong has started to identify nomological necessity's instantiation with causation in this explicit form as direct answer to van Fraassen's identification challenge.

Note that the identification problem can also be interpreted in an epistemic way: how do epistemic subjects identify, i.e., know about or perceive nomological necessity? Armstrong's suggestion emerges already from the quotes given above but here's an even more explicit statement: "The Identification problem is solved via our direct awareness, in certain favorable cases, of causation in the token case." (Armstrong 1997: 228; my italics) 
[interfering factor] H" (Armstrong 1997: 231; my addendum in square brackets). Translated into talk about events and causation the story about interference is the (almost) truism that it is always possible to interfere with a causal process that relates (spatio-)temporally distinct events. ${ }^{4}$

Taking these considerations into account, Armstrong distinguishes between "oaken" and "iron laws": $N(F, G)$ is iron just in case there is, as a matter of fact, no physically possible interference. $\mathrm{N}(\mathrm{F}, \mathrm{G})$ is an oaken law (in later publications Armstrong speaks of a "defeasible law") if there are possible interferers (cf. Armstrong 1997: 231).

Now, when focussing on the logics or semantics of interferences the mere addition of a proviso clause to the entailment postulation- "The entailment actually holds only for the case where it is given that nothing further interferes"-might resolve any trouble one could have with the subject of oaken laws or interferences and, so, Van Fraassen's inference problem could still count as settled. ${ }^{5}$

Yet, the difficulties start (latest) when we aim to conceptualise within Armstrong's metaphysical framework what happens in such interference cases. Especially puzzling will be the question what happens in such cases to nomological necessity and its instantiation. This is the question I address in this paper.

The difficulties I will unearth have not come to our attention yet because, or so I believe, Armstrong has tied the problem of inter-ference too closely to van Fraassen's problem of $i n$-ference, i.e., he has treated preventions and interferences mostly in terms of semantic issues of entailment or entailment provided that rather than on metaphysical grounds.

Whether my guess is true or not, I am sure that Armstrong would support the enterprise to address these issues also from an ontological, not only from a logical point of view, for he says elsewhere that we should not be "concerned with true statements of law, but rather with the truthmakers of such statements." (Armstrong 2004: 127).

As I said earlier, once we turn to the metaphysical issue we can treat Armstrong's theory as a sample case for all kinds of theories of lawhood that see a kind of necessity at work regarding law governed causal events in nature.

\section{The InTER-FERENCE Problem, Metaphysically CONSTRUEd}

I turn, hence, to the ontological problem. In the case of interferences we encounter the following situation:

- There is the atemporal second order state of affairs (the law) that $\mathrm{N}(\mathrm{F}, \mathrm{G})$.

- There is the first order state of affairs that, at a certain time and place $<x, y$, $\mathrm{z}, \mathrm{t}>$, something $a$ is $\mathrm{F}$.

- There is the interfering state of affairs at that time and place (or shortly afterwards at $\mathrm{t}+\varepsilon$ ) that $a$ is also $\mathrm{H}$.

However, or rather, consequently,

\footnotetext{
${ }^{4}$ Cf., famously, Bertrand Russell in (Russell 1912).

${ }^{5}$ I leave aside the vast literature on so called "ceteris paribus laws" that shows how controversial the semantic issue of proviso clauses really is (but compare Author 2007a). Also, I will not question whether van Fraassen's problem is, in fact, solved. My aim is to show that whether or not the issue of proviso clauses and the inference problem are resolvable semantically we still encounter grave metaphysical problems. These problems are the focus of my paper.
} 
- $\quad$ we do not have, at $\mathrm{t}$ (or a short time afterwards at $\mathrm{t}+\Delta \mathrm{t}$ with $\mathrm{t}<\mathrm{t}+\boldsymbol{\varepsilon}<\mathrm{t}+\Delta \mathrm{t}$ ), the state of affairs that $a$ is $G$.

The crucial question is, now: what happens to N? Does it instantiate together with F as it should if we follow the answer to van Fraassen's inference problem or not? The difficulty is that N's instantiation is supposed to be causation but causation exists only amongst actual events: $a$ causes $b$ only if both $a$ and $b$ are the case. In other words, without $\mathrm{G}$ occurring, $\mathrm{N}$ cannot be the causation of G: "we should deny that these are cases of causation [...] no relation exists without its full complement of terms." (Armstrong 1997: 75) ${ }^{6}$

Consequently, interference cases, interpreted from within Armstrong's metaphysics, seem to be internally incoherent:

(1) If $\mathrm{N}(\mathrm{F}, \mathrm{G})$ is a law then $\mathrm{N}$ instantiates whenever $\mathrm{F}$ instantiates (as answer to the inference problem).

(2) N's instantiation is causation, here: the causation of $G$ (to answer the identification problem)

(3) There are cases of prevention where some interferer prevents G from coming about although $\mathrm{F}$ is instantiated (= there are oaken laws).

(4) Causation demands success.

Contradiction: you cannot have $\mathrm{N}$ instantiating as causation whenever $\mathrm{F}$ instantiates while its effect, $G$, does sometimes not come about.

Our task for the rest of the paper is to establish which of the four claims we had better drop in order to re-establish coherence without distorting too much Armstrong's original theory of laws of nature. While (1)-(3) are principally up for grabs I do believe that (4) should be left untouched: it is impossible for F to cause $G$ (qua N's instantiation) and, yet, G does not happen (but see Fn. 7 if you have different intuitions).

Here are, then, ways in which (1)-(3) can be denied (prima facie at least):

(N1) $\mathrm{N}$ does not instantiate in cases of interference.

(N2) N does instantiate but not as causation (an alternative has to be given). ${ }^{7}$

(N3) No prevention is possible for real laws ( $=$ there are no interferences $=$ there are no oaken laws).

There are two versions of negation 3:

(N3.1) The firs t law-relatum is not what it seemed to be. N(F, G) has never been the law; instead, it is the strict $\mathrm{N}$ (uninterfered-with-F, G).

\footnotetext{
${ }^{6}$ In this quote Armstrong is not talking about interference cases but about probabilistic causation. Yet, the message that causation demands success is the same. Even Humean minded metaphysicians agree in this respect: "[only] causal dependence among actual events implies causation." (Lewis 1986: 167; my addendum and italics)

${ }^{7}$ If you believe that (4) above can be denied (contra my, Armstrong's and Lewis's intuitions) then we are not, after all, in major disagreement, for we could treat our dispute as mere verbal and agree that my negation 2 (that $\mathrm{N}$ instantiates as something else) is your negation of 4 . In other words, while you say that causation does not need success I say that you have something else rather than causation in mind which you happen to call "causation".
} 
(N3.2) The s e c o n d law-relatum is not what it seemed to be. N(F, G) has never been the law; instead, all laws have the form of the strict $\mathrm{N}\left(\mathrm{F}, \mathrm{D}_{\mathrm{G}}\right)$, where $\mathrm{D}_{\mathrm{G}}$ is (merely) a disposition to bring about G.

What unifies negations $(\mathrm{N} 2),(\mathrm{N} 3.1)$ and $(\mathrm{N} 3.2)$ is that each one of them alters one of the three elements that make up Armstrong laws: (N2) changes N, (N3.1) alters F, and (N3.2) replaces G. What relates negations $(\mathrm{N} 2)$ and $(\mathrm{N} 3.2)$ is that they both remove the success condition (this will become obvious only later).

In what follows, I will first have a look at $(\mathrm{N} 1)$ and reject this option as inadequate rather quickly. I then jump to (N3.1) which will turn out to be a more profound alteration of Armstrong's theory than it might seem at first sight. Afterwards, I discuss the thematically linked $\mathrm{N}(2)$ and (N3.2). Although they, too, will not prove to be entirely satisfactory they indicate the direction for a novel theory which I will introduce in the final section of the paper.

\section{(N1) N DOES NOT INSTANTIATE IN CASES OF INTERFERENCE}

Considering a quote from What is a law of Nature makes clear from the outset that (N1) can't be our solution to the problem of interferences:

If $\mathrm{F}$ and $\mathrm{G}$ are related by a dyadic relation $[\mathrm{N}$, that is] [...] then it cannot be that they have this relation at one time or place yet lack it at another. The universals $F$ and $G$ are exactly the same things at their different instantiations [...] If it holds in one instance then it holds in them all, because it is the one identical thing in all the instances. (Armstrong 1983: 79; my addendum in square brackets)

An even more convincing reason against (N1) is that endorsing it would weaken to an unacceptable extend Armstrong's general solution to van Fraassen's inference problem, where it was crucial that $\mathrm{F}$ does drag $\mathrm{N}$ along because of $\mathrm{N}(\mathrm{F}, \mathrm{G})$ : the answer to van Fraassen is only convincing if $\mathrm{F}$ brings $\mathrm{N}$ with it no matter what.

Note that (N1) is even less a possible solution for the problems of interference if Armstrong follows Baxter's suggestion (Baxter 2001) in postulating that "the connections between universals involved in laws of nature are [metaphysically] necessary rather than contingent" (Armstrong 2004: 136; my addendum in square brackets). Although this is a suggestion Armstrong has dropped again since it is worth noting that a metaphysically necessary link (instead of a mere nomologically necessary link) aggravates the problem: it simply makes no sense to say that a metaphysically necessary relation between universals could locally, where there's an interference, cease to hold.

One might be tempted, at this point, to introduce a further nomic relation next to $\mathrm{N}$, namely nomic exclusion (cf. Tooley 1977: 676, 679). Yet, nomic exclusion, $\mathrm{E}$, as originally conceived by Tooley, was meant to exclude $G$, not N, from coming about: $E(H, G)$. That is, Tooley's original nomic exclusion would not exactly deliver what we were looking for above. But even if we accept it as a new variant to deal with interference cases we face new challenges. We have, for example, to ask how nomic exclusions, E, and nomic necessitations, $N$, interact when both instantiate: suppose that we have $N(F, G)$ but also $\mathrm{E}(\mathrm{H}, \mathrm{G})$ where the latter means that $\mathrm{Hs}$ nomically exclude Gs. Also, suppose that Fa and Ha. Are there laws mediating between instantiated Ns and Es? Do Es always trump Ns or do both come in various strengths? Nomic exclusion, E, would also face a new identification problem: could it's instances, exclusions, also be causations of a sort? Moreover, N's own instantiation can still not be causation for it still does not always lead to success. ${ }^{8}$

\footnotetext{
${ }^{8}$ See similar considerations below in both sections on forces and force laws.
} 
In short, (N1) is unacceptable.

\section{(N3.1) N(F, G) IS NOT THE LAW; INSTEAD, THE STRICT N(UNINTER- FERED-WITH-F, G) IS.}

Maybe $\mathrm{N}(\mathrm{F}, \mathrm{G})$ is not really the law we should be looking at in the first place (it has actually never been a law) but rather something like $\mathrm{N}$ (uninterfered-with-F, $\mathrm{G}$ ). That is, our first relatum, F, is of a different nature than we have envisaged so far. Armstrong mentions this option explicitly elsewhere: "But cannot an oaken law [a law with possible interferences] always be represented, in principle at least, as an iron law [a law where no prevention is possible] by putting in all the negative qualifications?" (Armstrong 1983: 149; my italics and addenda). He gives an affirmative answer:

Yes, in a way it can, provided that we bear in mind how wide the qualifications may be which are implied by the phrase 'in principle'. It could even be that the statement [...] would have to be of infinite length. (Armstrong 1983: 149) ${ }^{9}$

The idea behind (N3.1) is, again, to claim that the nomological state of affairs that constitutes the law has never been $\mathrm{N}(\mathrm{F}, \mathrm{G})$ but rather something like $\mathrm{N}$ (uninterferedwith-F, G). If this strategy proves to be successful we have effectively denied entry (3) of the above multi-lemma: any causal process governed by real laws is such that there is no interference possible.

Although this solution looks quite elegant it, too, has its difficulties. I will discuss three such problems, (i) to (iii). They all relate to the challenge to spell out what universals like uninterfered-with-Fs exactly are. On our way to a characterisation we will see that, in order to fulfil their required role, (i), uninterfered-with-Fs must be enormously complex, (ii), their instantiation must encompass spatio-temporally extended regions, (iii), they must be very numerous. All this might count against the existence of these universals, or against the laws they figure in, or both.

(i) For a start, note that if uninterfered-with-Fs were something like $\neg \mathrm{H} \wedge . . \wedge \neg \neg \wedge \mathrm{F}$ we would be confronted with negative universals which many metaphysicians, including Armstrong, reject. Luckily, there could be a way to circumvent this problem: we might be able to stipulate that there is a positive, yet, complex, structural universal that, when instantiated, fixes the sum total of all (positive) states of affairs at least for a sufficiently large region in space so that interferers are excluded simply because such a complex state of affairs includes and fixes all there is and, by fiat, all there is has no interferer. ${ }^{10}$

This will remind readers of Armstrong's Truth and Truthmakers of totality facts as truthmakers for general and negative truths (cf. Armstrong 2004: 68-82): the totality of

\footnotetext{
9 Although I will now discuss this idea as a prima facie possibility it has to be noted that the way it is presented in Armstrong's quote is slightly at odds with the project he is generally engaged with. Let me explain. Instead of "cannot an oaken law always be represented as an iron law" the question should rather be: "is, what seemed to be an oaken law, not in reality an insufficiently well represented iron law?" or, again in other words, "are what we have called 'oaken laws' not only rough and ready incomplete approximations to the real laws"? After all, Armstrong's aim is to give a metaphysical theory of what laws of nature are and not how fallible epistemic subjects represent the laws. Therefore, if a so called "oaken law" can be "represented" as an iron law then the "oaken law" has never been a law in the first place. Rather, the, as yet, misrepresented or undiscovered iron law is the law.

${ }^{10}$ Independent of Armstrong's theory one could allow negative universals and so, maybe, make the above amendments to the first relatum less complex. I say "maybe" because a vast number of interferences must fail to occur and, thus, all those failings have to be mentioned. Anyway, this is a path well worth pursuing further but limited space does not allow for this in this paper.
} 
first-order positive states of affairs plus the higher order state of affairs (the totality fact) that these are all the first-order states of affairs makes true certain general propositions and also negative statements about the first-order positive states of affairs. It is along these lines that I believe one could probably capture what uninterfered-with-Fs are.

(ii) The required complex totality fact types have to be expanded in yet another dimension for we have not yet considered time: there are cases of late preventions where $\mathrm{H}$ comes about after $\mathrm{F}$. In other words, while an $\mathrm{F}$ might have been instantiated without any interferer at $t$, it could be interfered with at $t+\varepsilon$ where $t+\varepsilon$ is still early enough to prevent $\mathrm{G}$ at $\mathrm{t}+\Delta \mathrm{t}$ (with $\mathrm{t}<\mathrm{t}+\varepsilon<\mathrm{t}+\Delta \mathrm{t}$ ).

But never mind, we have already extended instantiations of Fs in space (in (i) above), so why not also in time? In response to late preventions, we can reply that when we talk loosely about uninterfered-with-Fs what we really mean is state of affairs types that instantiate as fairly large four dimensional events. These 4D worms are temporally extended at least until an infinitely short time just before the instantiation of G.

(iii) Yet, even if we accept these four dimensional totality fact types as uninterfered with-Fs we are not at the end of the story for there is presumably more than one such type of super state of affairs that incorporates $F$ and that leads to a $G$. That is, not only is the law's first relatum much more complex and encompassing than we thought, but what seemed to have been just one law, N(uninterfered-with-F, G), actually emerges as a cluster of a huge number of nomological relations each connecting one such super state of affairs type (including F) with G: Fs are Gs is a law iff $\{\mathrm{N}$ (uninterfered-with-F*, G), $\mathrm{N}$ (uninterfered-with- $\left.\mathrm{F}^{* *}, \mathrm{G}\right), \mathrm{N}$ (uninterfered-with-F***, G)...\}. ${ }^{11}$

Suppose we managed to take care of (i) to (iii) in a satisfying way. Just how far have we departed from what was originally supposed to be a law of nature, namely a relation between simple, non-structural, and probably fundamental universals? We have made two major changes: (a) laws are, now, clusters of relations and not merely a single relation, (b) $\mathrm{N}$ does not relate atomic, universals anymore (masses and charges to forces or accelerations) but hyper-complex four-dimensional structure types. Maybe this is a revision one could happily accept ${ }^{12}$ but it is certainly a radical change from our orthodox picture of lawhood, both pre-theoretical and of what is generally perceived to be Armstrong's theory.

This becomes even more obvious if we start to wonder what has happened to the original simple universal $\mathrm{F}$ within these super state of affairs types: the original $\mathrm{F}$ is now degraded to an INUS condition for the antecedents of members of the respective law cluster: an F becomes an insufficient but necessary part of an unnecessary but sufficient super state of affairs type which stands in the relation of nomic necessity to G. I take it that this is not quite what we have in mind when we say that it is a law that masses attract or that electrons repel each other.

We can also ask whether the role that $\mathrm{N}$ and its instantiation was supposed to play has changed. One thing is sure, there is no reason for $\mathrm{N}$ not to instantiate whenever the

\footnotetext{
11 Armstrong is not unaware of the difficulties discussed in (i)-(iii). In fact, before he develops his theory of laws of nature in detail in his What is a Law of Nature (1983) and further in A World of states of Affairs (1997), he already discussed the need for the expansion of the first relatum of nomological necessity in his A Theory of Universals (cf. Armstrong 1978: 154-6). Yet, because his fully developed theory of laws comes afterwards he did not discuss the effect this need for expansion has on his later theory. This is what I will do next.

12 As Armstrong did at one point: "It is then suggested that when we consider all such potential interferences, interference with interference, etc., the resulting regresses cannot stop short of the whole state of the universe at that time. Only then do we reach the total cause." (Armstrong 1978: 155) Today, however, he prefers a different solution (see the discussion under (N2)).
} 
right hyper complex four-dimensional structure type instantiates. Yet, would we still call such an $\mathrm{N}$ instantiation "causation"? The trouble is not that its effect might not occur (for it definitely does) ${ }^{13}$ but rather that N's instantiation loses its locality. We are not any longer looking at a single object at a particular space-time point and a single universal, the former F, that causes $G$ but, here, N's operation is spread out over four dimensional space-time-areas. $\mathrm{N}$ becomes a (mysterious) holistic global matter. I say "mysterious" because, as a consequence of N's being spread out and global, its instantiation's observability (i.e., causation's observability) is at stake. ${ }^{14}$

So, hasn't (N3.1) it all wrong? If we are willing to reduce the former antecedent universals (the Fs) to INUS conditions (which is an otherwise respectable view of causes) and let causation spread out over large space-time areas (again something some people are willing to accept as a consequence of possible interferences) then (N3.1) is not entirely disreputable.

There is, of course, an epistemic problem lurking around the corner here: knowing the laws - these clusters of $\mathrm{N}$ relations amongst vast structural universals-is impossible for finite epistemic subjects like us. We can only guess at them and write down approximations like $\mathrm{N}(\mathrm{F}, \mathrm{G})$ for our day to day use. Whether this epistemic issue counts against the metaphysics of laws I do not want to judge.

However, apart from that, one might share the intuition (as I do) that complexity should result from the (incidental) interplay of many individual nomological factors rather than that nomicity arises from complexity. There is a strong intuition that it is the components that give rise to the whole and not the whole that leaves at best some secondary importance to its parts. It is, for this reason, that I won't pursue (N3.1) any further for the time being (but I will indicate a possible twist at the very end of the paper). For now, I search for a theory that both meets the locality intuition and solves the difficulties with interferences.

\section{INTERMEDIATE CONCLUSION}

Let me summarise. Armstrong's theory of laws of nature confronts us with difficulties when it comes to interferences with or preventions of law-governed causal processes. N, the law maker, which holds as a second order relation between universals (for example, $\mathrm{N}(\mathrm{F}, \mathrm{G})$ ), is supposed to instantiate as causation (of $\mathrm{G}$ ) whenever the first relatum, $\mathrm{F}$, is instantiated. Yet, in prevention cases the effect $\mathrm{G}$ might not come about so that either (N1) also N should not instantiate or (N2) N's instantiation can't be causation, for causation demands success, or (N3) it is wrong in the first place to assume that $\mathrm{N}(\mathrm{F}$, $\mathrm{G}$ ) is the law (rather, something else is, which is free from possible preventions).

We have, so far, tried to resolve the tension in Armstrong's theory by saying that $\mathrm{N}$ does, indeed, not instantiate in interference cases, (N1) (this option was rejected), and by reformulating the initial law so that interferences cannot cause trouble by fiat, (N3.1). This option might be open but reasons to look for a different solution, the locality of causation and an epistemic concern, were given.

It is worth noting that the mentioned difficulties are by no means only a problem for Armstrong. In fact, any theory of laws which binds causal relata together by a kind of necessity will have to face them: suppose you think that what makes Fs are Gs a law is that $\square \forall \mathrm{x}(\mathrm{Fx} \supset \mathrm{Gx}$ ) (where the box stands for metaphysical necessity) then you also

\footnotetext{
13 "Each actual total cause will nomically necessitate its effect.” (Armstrong 1978: 156)

14 This claim might be countered by pointing out that we can experience something by experiencing a part of it. (Thanks to an anonymous referee for pointing this out to me.)
} 
face the problem that Fs, when interfered with, might occur without G. However, nothing should be able to interfere with metaphysical necessity and, thus, the same machinery of F-alterations, addenda, and expansions like above would have to be applied. ${ }^{15}$

\section{INTERLUDE: THE (FUNDAMENTAL) FORCE LAWS}

There are two further possibilities left to discuss of how we could resolve the tension in Armstrong's theory. Both give individual, simple universals back the power they had lost to the clusters of global states in (N3.1): we could, (N2), try to find a different kind of instantiation for $\mathrm{N}$ (instead of causation) that allows $\mathrm{N}$ to be unsuccessfully instantiated. In other words, we could be looking for an appropriate middle ground for $\mathrm{N}$ somewhere in between not being instantiated and being instantiated as causation. That is, we could search for a "softer" $\mathrm{N}$ that is not quite causation but more than nothing.

Alternatively, (N3.2), we could give G a new interpretation that would allow it to be instantiated no matter what, i.e., even in interference cases: $\mathrm{N}$ could strictly bestow on Fs the potential to be or become $G$. That is, rather than $\mathrm{N}(\mathrm{F}, \mathrm{G})$ our laws could have the form $\mathrm{N}\left(\mathrm{F}, \mathrm{D}_{\mathrm{G}}\right)$, where $\mathrm{D}_{\mathrm{G}}$ is (merely) a disposition or potential to be or to become $\mathrm{G}$.

Focussing our attention for a while on a special kind of law-the (fundamental) force laws - we get some inspiration for both (N2) and (N3.2). Armstrong himself takes force laws as one of his prime examples when talking about laws with interferences. (In fact, I believe that force laws have been the model for the entire theory of nomological necessity but this is just a speculation.)

An important general feature we ordinarily, in day-to-day intuitions, associate with forces and which will come in handy for later purposes is that forces push to a certain degree in a specific direction whether or not they successfully accelerate or deform the object they operate on. Provided no other forces are active success is guaranteed. If, however, other forces counteract we only get a smaller acceleration (or deformation) according to the resultant force (which might well be zero). Realism about component forces assumed, ${ }^{16}$ we can stipulate that forces are always instantiated whether or not they successfully bring about the respective acceleration. Note that the superposition of forces is well understood. Here, interference is quantifiable and calculable. In fact, every schoolgirl and -boy learns about the basic vector algebra of force addition. In other words, interference and overlap within the realm of forces poses no problem.

In the following discussions of (N2) and (N3.2), I will suppose that either N, nomological necessity, or $\mathrm{G}$, the law's second relatum is, in the relevant sense, like a force. This idea seems to have some potential to solve the problem of interference.

In the final section to this paper, I will focus on force laws only and explore whether Armstrong's theory (and similar necessity based theories), when limited solely to these laws, draws a coherent picture. Force laws, so it seems, can handle interferences with relative ease.

\footnotetext{
15 In (Author 2010a\&b) I particularly target dispositional essentialists (esp. (Ellis 2001) and (Bird 2007)), who utilise metaphysical necessity where nomological necessity figures in Armstrong.

${ }^{16}$ I will defend component-force realism in the final section.
} 


\section{(N2) N DOES INSTANTIATE BUT NOT AS CAUSATION}

So, let N's instantiation (whenever $F$ is instantiated) not be the causation of $G$ but rather a kind of force or forceful push towards G. Only if there are no other such pushes or influences due to further features of the situation (the object being $\mathrm{H}$ as well as F, for example) will $G$ really be brought about. Otherwise $G$ is not (or only partially) instantiated.

Armstrong sympathises with this move. He believes that (provided my arguments are sound so far) N's instantiation could be a kind of factor of causation. The totality of all factors operating at one place is, then, the causation in that instant. ${ }^{17}$ However, according to Armstrong, what the nature of these factors is remains to be seen. And, indeed, as shall be revealed now, my reference to forces is at best metaphorical.

Here is why: for (Newtonian) forces we have a (vector) algebra readily available and, so, know exactly how to calculate the influence of interfering forces. Specifically, we know what it means for a force to be cancelled out by another force (both forces acting upon the same object with the same strength, yet, in exactly opposite directions).

Unfortunately, we do not have any such algebra or theory available for Ns and arbitrary causes. We could, of course, leave our theory at a metaphorical level and only hand-wavingly gesture at forces but I fear only a few metaphysicians would find that satisfying. ${ }^{18}$ Yet, once we start to form a theory we seem to face very similar problems to those we have already encountered in (N1) and our interlude on Tooley's theory which introduced nomic exclusion as a second nomological factor: presumably, the interaction of two different $\mathrm{Ns}$ - the one that $\mathrm{F}$ brings with it and the one the interferer $\mathrm{H}$ brings with it - is regular, even lawlike, and not just so happening. Consequently, we would need "a new N which gives the rule (law) for summing the factors" (Armstrong 2008) ${ }^{17}$. This is problematic for many reasons.

First, note that we are, then, confronted with a reiteration of nomicities: there are first level Ns, call them " $\mathrm{N}$ ", which instantiate as force-like factors of causation and there are, additionally, second level Ns, call them " $\mathrm{N}^{2}$ ", that mediate between those factors. Second, we would have to answer the question whether those second level $\mathrm{N}^{2} \mathrm{~s}$ form strict laws. If we cannot argue convincingly that they do we end up in an infinite regress (for we'd need mediating $\mathrm{N}^{3}$ s...). Third, van Fraassen's identification problem has its bite again: what is $\mathrm{N}^{2}$ ? 19

Now, in an Ockhamian mood one might want to deny that $\mathrm{N}^{2}$ is a new $\mathrm{N}$ and postulate that $\mathrm{N}^{2}=\mathrm{N}^{1}=\mathrm{N}$. This is not satisfying either, for if $\mathrm{N}^{1}$ and $\mathrm{N}^{2}$ are the same so are their instantiations. Yet, it seems odd to say that sometimes $\mathrm{N}$ instantiates as a factor of causation of events and sometimes as a mediator between those causal factors.

Maybe there is a way out of these difficulties but it seems that any solution will metaphysically be a little speculative and contrived. ${ }^{20}$ I therefore reject also option (N2).

\footnotetext{
${ }^{17}$ Personal communication, February 2008.

18 See, however, Stephen Mumford and Rani Lill Anjum's promising vector theory of dispositional causation (Mumford \& Lill Anjum 2011) which bears some similarities to (N2).

19 As a referee of this journal pointed out, the second problem is an empirical issue and, admittedly, it might turn out in our favour. Yet, it also might not.

${ }^{20}$ However, Richard Corry (Corry 2006), coming from a different non-Armstrongian angle to the same issue, formulates a convincing theory very much like (N2).
} 


\section{(N3.2) THE SECOND LAW-RELATUM IS NOT WHAT IT SEEMED TO BE. INSTEAD OF $G$ WE HAVE THE DISPOSITION D $_{G}$}

I am afraid, we do not gain much by transferring the force character from $\mathrm{N}$ into the second relatum and replace $\mathrm{G}$ in $\mathrm{N}(\mathrm{F}, \mathrm{G})$ by the (maybe basic, unanalysable) power $\mathrm{D}_{\mathrm{G}}$ to bring about $\mathrm{G}$. In fact, we are merely shifting most of the metaphysical burdens $\mathrm{N}$ had to carry in $(\mathrm{N} 2)$ onto $\mathrm{G}$ or, now, $\mathrm{D}_{\mathrm{G}} \cdot{ }^{21}$

Here's first a description of the picture we get in (N3.2): just as in (N2), N itself instantiates whenever F does. However, contrary to $(\mathrm{N} 2)$ here also $\mathrm{D}_{\mathrm{G}}$ instantiates. Yet, $\mathrm{D}_{\mathrm{G}}$, while surely present whenever $\mathrm{F}$ is, is (merely) a dispositional property which, while present, might not manifest itself (i.e., $D_{G}$ does not have to bring about $G$ ). Yet, what seems first to be a solution to the interference problem turns out, on closer inspection, to be a veiling strategy. Our problems have not disappeared, they merely hide under the cover of $\mathrm{D}_{\mathrm{G}}$.

Note the parallels to (N2) above: unless we leave the talk about the disposition or power $\mathrm{D}_{\mathrm{G}}$ at a rather abstract level we must answer, also for dispositions just as much as for the $\mathrm{N}$ in (N2), what exactly happens in interference cases. Are there G-preventing as much as $\mathrm{G}$-causing powers, i.e., $\mathrm{D}_{\mathrm{G}-\text { versus }} \mathrm{D}_{\mathrm{G}+}$ ? Is there a calculus telling us how to combine $\mathrm{D}_{\mathrm{G}-}$ and $\mathrm{D}_{\mathrm{G}+}$ ? Are there laws governing the interplay of $\mathrm{D}_{\mathrm{G}-}$ and $\mathrm{D}_{\mathrm{G}+}$ ?22

Moreover, we might want to ask what the connection between the disposition $\mathrm{D}_{\mathrm{G}+}$ (and, similarly, $\mathrm{D}_{\mathrm{G}-\text { ) }}$ and $\mathrm{G}$ is itself? A metaphysically necessary entailment would not be in the spirit of Armstrong's theory. And a further law of nature? If so, it would itself have to be a defeasible law since the whole reason why $\mathrm{D}_{\mathrm{G}^{+}}\left(\mathrm{D}_{\mathrm{G}}\right)$ was introduced in the first place was because something might have the disposition to be $G$ without actually being $G$. So we would need an oaken law relating $D_{G+}$ to $G$ and we seem to be on the first step of an infinite regress. ${ }^{23}$

Finally, it has to be mentioned that Armstrong would rather not allow dispositions into his ontology: "We should not postulate any [...] properties and relations (universals) save actual, or categorical, properties and relations" (Armstrong 1983: 8-9; my emphasis; cf. also Armstrong 1997: 261).

I believe we are in the same or a similar metaphysical predicament as in (N2). When trying to work out the details of a theory along (N2) or (N3.2) Armstrong or the Armstrongian would be confronted with unexpected ontological complexity. I therefore also reject (N3.2).

As I have indicated, the difficulties for (N2), N(3.1) and (N3.2) are equally present for theories of lawhood that see necessities other than (Armstrong's) nomological necessity as lawmaker, for, clearly, no binding force worthy of the name "necessity" can allow for interferences. The plausible means of resolving the difficulties we have collected while

\footnotetext{
${ }^{21}$ Some philosophers have pointed out that the problem of interference and prevention is, in the guise of so called "ceteris paribus laws", also a grave problem for regularity theories of laws (cf. (Hüttemann 1998), (Lipton 1999), (Kistler 2003), and Cartwright in various publications, for example (Cartwright 1992)). In fact, some even claim that one can see regularity theories fail because of ceteris paribus laws. These philosophers offer dispositionalist accounts of laws of nature as an alternative where, just like in $\mathrm{N}(3.2)$, laws correlate properties with their powers to bring about events rather than that laws state event regularities. For an analysis of these accounts and why they are also problematic see (Author 2007b).

${ }^{22}$ Here (N3.2) might have the advantage over (N2) in that it would not involve iterations of Ns. Moreover, on the level of dispositions, i.e., first order properties, rather than on the level of $\mathrm{N}$, these questions could turn out to be broadly up to science and future philosophy to answer.
}

${ }^{23}$ Thanks are due to an anonymous referee who pointed this further problematic issue out to me. 
discussing Armstrong are, therefore, also means to be adjust similar necessity approaches to laws.

\title{
FORCE LAWS TO THE RESCUE!
}

Is there another way out? Above, I have praised a particular kind of laws, the force laws, which, so it seems, can handle interferences with relative ease. In the next section, I will focus on the force laws only and explore whether Armstrong's theory, when solely projected onto these laws, draws a coherent picture.

Armstrong writes regarding the law of gravitation and possible interferences:

\begin{abstract}
The gravitational laws give the gravitational forces holding between two bodies having certain masses and a certain distance from each other. It is not necessary that these forces cause the two bodies to move towards each other. There may be many other bodies also exerting gravitational force in the situation, not to mention other types of forces [...] that may be operating. [...] We can never rule out the possibility, mere possibility though it may be, that further forces [...] could be added to the situation which would alter the behaviour of the particulars involved. (Armstrong 1997: 230-1)
\end{abstract}

How do we formalise the law of gravitation within Armstrong's original framework? The first sentence-_"The gravitational laws give the gravitational forces holding between two bodies having certain masses and a certain distance from each other."-- suggests that $\mathrm{N}$ holds between the structural state of affairs type "two bodies having certain masses, $\mathrm{M}_{1}$ and $\mathrm{M}_{2}$, and being at a certain distance, $\mathrm{D}$, from each other" and the gravitational force, $F_{g}$. That is, the law of gravitation reads: $N\left(M_{1} \wedge M_{2} \wedge D, F_{g}\right)$. Note that this interpretation is akin to the general interpretation (N3.2) where we have replaced $G$ by a disposition $D_{G}$ to bring about $G$. Here, we have a force $F_{g}$ which might or might not cause an acceleration $A$.

There is a second plausible interpretation of the force laws. It follows interpretation (N2) from above: the law of gravitation could also state that bodies accelerate with acceleration A towards each other if they have certain masses and are at a certain distance: $N\left(M_{1} \wedge M_{2} \wedge D, A\right)$. Where has the gravitational force gone in this picture? The answer is obvious when we compare to (N2): we identify N's instantiation with the force. I will come later to this promising interpretation, call it "(Force)", and start first with $\mathrm{N}\left(\mathrm{M}_{1} \wedge \mathrm{M}_{2} \wedge \mathrm{D}, \mathrm{F}_{\mathrm{G}}\right)$, which I will refer to as "(Proto-Force)".

Needless to say, in (Proto-Force), all other force laws ${ }^{24}$ - for example, Coulomb's law: $\mathrm{N}\left(\mathrm{Q}_{1} \wedge \mathrm{Q}_{2} \wedge \mathrm{D}, \mathrm{F}_{\mathrm{c}}\right)$ - have to be interpreted in very much the same way. The great advantage of this interpretation is that we are able to claim that all those laws are iron laws in the sense that no interference is possible: that a massive particle might also be charged does not take away the gravitational force. Rather, Coulomb's force acts as well but that does not annihilate the first force. Coulomb's force might enforce or reduce (even to zero) the gravitational force's effect, but it won't make the gravitational force itself disappear and it is this force, not the force's effect, which is the law's second relatum. ${ }^{25}$

For forces, unlike any general $\mathrm{D}_{\mathrm{G}}$ as in $(\mathrm{N} 2)$, we have an algebra readily available that tells us the rule or law by which a multitude of overlapping and interfering forces will interact. As a consequence, we can hope to be able to add to the individual force laws, (i), a further law governing the interaction of a multitude of different forces, and, (ii), a law

\footnotetext{
${ }^{24}$ If present physics is correct these are, next to the gravitational force, the electro-magnetic force and the weak and strong nuclear forces.

${ }^{25}$ I will give further arguments for the here assumed reality of component force later.
} 
mediating between resultant forces and accelerations. The first law, (i), says that all individual forces operating on a certain body with mass $\mathrm{M}$ add up by means of vector addition: $\mathrm{N}\left(\mathrm{F}_{1} \wedge \ldots \wedge \mathrm{F}_{\mathrm{n}} ; \mathrm{F}_{\mathrm{T}}=\sum \mathrm{F}_{\mathrm{i}}\right)$ (the $\mathrm{F}_{\mathrm{i}}$ being vectors). ${ }^{26}$ The second, (ii), tells us that the so calculated total or resulting force, $\mathrm{F}_{\mathrm{T}}$, operating on an object with mass $\mathrm{M}$ causes it to accelerate with $\mathrm{A}=\mathrm{F}_{\mathrm{T}} / \mathrm{M}$, that is $\mathrm{N}\left(\mathrm{F}_{\mathrm{T}} \wedge \mathrm{M}, \mathrm{A}=\mathrm{F}_{\mathrm{T}} / \mathrm{M}\right)$. Note that both laws are perfectly strict and uninterferable: no matter what, the total force results from vector addition of all component forces and the acceleration of an object is certain considering the total force acting upon it (as long as $\mathrm{F}_{\mathrm{T}}$ really remains the total force). In short, it looks as if, for the force laws, we get rid altogether of the problems of interferences and preventions. (So, item (3) in our original list of together incoherent claims can successfully be denied.)

Yet, there's one drawback for this interpretation (the discussion of which will smoothly lead to our second interpretation of the force laws, (Force)). N's instantiation is secured because there is no interference, yet, can it be conceived of as causation? Do masses and distances "cause" the forces between them? Do component forces "cause" resultant forces? If we have a conception of causation where causation is a "bringing about of events" (and many, including Armstrong, have this push-and-pull intuition of causation) then the notion of causation has to be stretched here a little. Especially when Armstrong states that causation is observable he relies on this picture of causation:

The dyadic predicate 'causes' is as much an observational predicate as any other predicate in our language, especially in such cases as our awareness of pressure on our own body. (Armstrong 1997: 228; my italics)

Also:

The philosophically little-discussed perception of pressure on our body—in general the action of force on our body -is as directly perceived, I maintain, as anything else in our experience. (Armstrong 2004: 128; my italics)

Yet, if masses in a distance "cause" a force between them-as interpretation (ProtoForce), namely, $\mathrm{N}\left(\mathrm{M}_{1} \wedge \mathrm{M}_{2} \wedge \mathrm{D}, \mathrm{F}_{\mathrm{G}}\right)$ would have it-than this causation is not directly perceived as pressure on our body for, simply, the force we do in fact feel on our body is not the force of $\mathrm{N}$ but the (Newtonian) force the presence of which $\mathrm{N}$ ensures.

When talking about the observability of causation Armstrong is, I believe, tacitly moving from interpretation (Proto-Force) to interpretation (Force), for remember that in the latter interpretation $\mathrm{N}$ itself instantiates as the indeed perceivable (Newtonian) force: (Force) says that the proper formalisation of the law of gravitation within Armstrong's framework is: $N\left(M_{1} \wedge M_{2} \wedge D, A_{G}\right)$. That is to say that bodies accelerate with $A_{G}$ towards each other if the two bodies have masses $\mathrm{M}_{1}$ and $\mathrm{M}_{2}$ and are at a distance $\mathrm{D}$ from each other. It is N's instantiation, namely a (gravitational) force, that brings this movement about (if no other force interferes). ${ }^{27}$

Yet, while (Force) is more in line with Armstrong's claim that N's instantiation is directly observable as pressure on our body it differs in other respects from Armstrong's

\footnotetext{
${ }^{26}$ As in (N3.1) where we tried to amend the laws first relatum, F, we might have to talk about totality facts: $\mathrm{F}_{1} \wedge \ldots \wedge \mathrm{F}_{\mathrm{n}}$ have to be all the forces there are. This, I hope, would be reasonably unproblematic here because we have limited our inquiry to forces, i.e., there is really just one type of entity to be considered.

${ }^{27}$ A similar suggestion has been made by Bigelow and Pargetter where forces are said to be at the heart of causation. Since Bigelow and Pargetter are not directly concerned with nomicity but with causation their suggestion is not quite the one proposed here, namely to replace Armstrong's $\mathrm{N}$ by fundamental forces. However, the features they attribute to forces are very similar to those Armstrong assigns to $\mathrm{N}$ : for them, forces are second order relations amongst events and their properties (conceived of as universals) (cf. Bigelow \& Pargetter 1990: 106ff.)
} 
original theory: if N's instantiation is a gravitational force (or an electromagnetic, weak or strong force) then $\mathrm{N}$, the second order relational universal, instantiates as a vectorial magnitude with both a direction and a strength. Moreover, the strength and direction of N's instantiation would be a function of N's relata. These might be alterations that could be accommodated but there are also some consequences of (Force) which are harder to deal with:

The most pressing one is how we explain within (Force) the nomicity of force interactions. As pointed out in (Proto-Force), force interactions themselves are lawlike: they universally obey vector additions. Yet, if we consult the respective formulations of these interaction laws within Armstrong's theory we see the problem immediately: both $\mathrm{N}\left(\mathrm{F}_{1} \wedge \ldots \wedge \mathrm{F}_{\mathrm{n}} ; \mathrm{F}_{\mathrm{T}}=\sum \mathrm{F}_{\mathrm{i}}\right)$ and $\mathrm{N}\left(\mathrm{F}_{\mathrm{T}} \wedge \mathrm{M}, \mathrm{A}=\mathrm{F}_{\mathrm{T}} / \mathrm{M}\right)$ would confront us with iterated nomological necessities for note that forces, the $\mathrm{F}_{\mathrm{i}} \mathrm{s}$, really are N's instantiations. This leads ultimately to the unacceptable oddity that forces force forces to interact. ${ }^{28}$

\section{Outline of A Positive Theory}

I now propose a solution to this riddle that deviates from Armstrong's grand nomological project. We could, so the idea, claim that interaction laws are mere means to tame swarms of forces, i.e., to calculate and to predict their overall effect. There's no further nomological necessity involved here since the whole (ontological) work is done already by the components. The desired link in nature that binds the world together has already been secured by nomological necessity's primary force instantiation. ${ }^{29}$

The obvious naturalistic advantage is, here, that forces can not only be observed as pressure on our bodies they can also be measured with instruments. Yet, there are also challenges to be met: (i) Some people reject a component forces realism while the account here presented is essentially realistic. (ii) Not all physical laws are force laws and, (iii), not all laws are physical laws. Moreover, even if there is an answer to (i)-(iii) we are confronted with, (iv), the threat that forces are no longer en vogue in present day physics.

To (i). A reason to reject the reality of component forces is the threat of overdetermination: if also resultant forces are real both the components and their resultant cause the acceleration (or deformation). Of the two obvious possibilities to avoid this problem the one to choose is, for our purposes, to grant reality solely to the components (as opposed to solely to the resultant). Only this strategy is in line with theory (Force) which claims that interaction laws are mere means to epistemically handle the interplay of component forces. The whole (metaphysical) work is done by these components.

However, some philosophers have claimed the opposite pro resultants and contra components (cf., for example, Cartwright 1983: 59). While I cannot conclusively prove here that we must vote for the components I can provide one or two further extrinsic motives in their favour. (The reason cited already above is internal to theory: the reality of components coheres best with (Force).)

For a start, it is valuable to note that the difference between component and resultant acceleration vectors can be deflated. As Alastair Wilson argues,

\footnotetext{
${ }^{28}$ Note aside that there's also an issue about types and tokens here: nomological necessity is the type of which forces are the token. I.e., for the above laws I should have written: $N\left(N_{1} \wedge \ldots \wedge N_{n} ; F_{T}=\sum N_{i}\right)$ and $\mathrm{N}\left(\mathrm{N}_{\mathrm{T}} \wedge \mathrm{M}, \mathrm{A}=\mathrm{N}_{\mathrm{T}} / \mathrm{M}\right)$ which makes the trouble even more obvious.

${ }^{29}$ Not coming from within an Armstrongian framework but focussing solely on component forces and their resultants, Lewis Creary (1981: 152) tells a similar story about the reality of components and the mere "explanatory" character of the only "mathematical" (i.e., not real) resultant forces.
} 
being a component acceleration vector and being a resultant acceleration vector are both frame-dependent properties of properties. The very same property will appear to be a resultant acceleration vector in one frame, and a component acceleration vector in others. It follows that the distinction between component and resultant acceleration vectors cannot support the ontological weight placed on it by sceptics about contributions; if we accept the reality of resultant vectors but not of component vectors, then what exists becomes a frame-dependent matter. (Wilson A. 2009: 597)

Next, we can point out that despite this deflation relative to reference frames, it is still possible to trace each (resultant) force back to its source(s) and we ultimately end up with a (finite) number of components that directly originate from charges, masses, or quark colour. That is, even if no acceleration vector can, frame independently, be singled out as more real than any other, we have an argument from the origin of forces that allows us to prioritise these (ultimate) components.

A further intuitive reason for component reality is that there seems to be a difference between a situation where two components add to zero (two horses, one pulling on each of your arms with an equal but not yet destructive force) to a situation where no such forces (horses) are present: in neither case will you move in either direction and, yet, you would surely prefer the latter to the former. ${ }^{30}$

To (ii). There are laws in fundamental physics which have prima facie nothing to do with forces. Examples are Heisenberg's uncertainty principle or Pauli's exclusion principle. Bigelow and Pargetter suggest that in cases like these "what happens is close enough to the actions of forces, as we conceive them" (Bigelow \& Pargetter 1990: 109). That is, in the case of Heisenberg we might want to say that a measurement of position disturbs the momentum, i.e., "forces" the momentum to be different. For Pauli, one might flirt with a statement like "it is impossible to force two identical fermions to occupy simultaneously the same quantum state".

Yet, if this talk were to remain a mere metaphor it would be dissatisfying as a solution to the above challenge. Luckily, Pauli's principle, for example, is partly responsible for the macroscopic effect that two solid objects cannot be in the same place at the same time and, thus, it indeed links to phenomena like solidity or impenetrability which are related to forces. The hope is that similar arguments can supplement the figurative speech in other cases in order to bridge the gap to more literal force talk. This, I fear, remains a task for future research.

To (iii). Where do the force laws stand in the larger scheme of things, i.e., if we take chemical, biological, maybe even psychological "laws" into consideration? I believe that a forces based theory of fundamental laws is not much worse of than any other such theory with naturalistic/reductionistic intentions (for example Lewis's). As Armstrong likes to point out: "It also rains on the enemy", that is, (Force) has no less and no more difficulties to explain how the living world or the mental world supervenes on the physical. Sure, if you deny any such supervenience (or reduction, or emergence) and believe in autonomous realms beside the physical then force laws can only be a small fraction of all laws.

To (iv). Even if we suppose that we can solve (i)-(iii) we still have to deal with the allegation that forces are no longer respectable entities in the physical sciences themselves. On the contrary, it occasionally seems that fundamental physics has abandoned talk about forces entirely. In macroscopic physics energy-based accounts (Lagrangians and Hamiltonians) replace forces, in the General Theory of Relativity geometry replaces forces, and quantum phenomena are best described in terms of

\footnotetext{
${ }^{30}$ Further support for component forces comes from (Bigelow and Pargetter 1990: 107-10), (Creary 1981), (Corry 2006), (Massin 2009), and (Molnar 2003: 194-198).
} 
probabilistic functions of initial conditions. Forces, one might radically conclude, have been deleted from scientific ontology. Like phlogiston they have had their day. If so, the rug is pulled out from under our feet: an (Armstrongian) account of force laws would be as useful as an (Armstrongian) account of witchcraft.

Yet, there's a growing community of philosophers who defend forces against this allegation. ${ }^{31}$ Here are the two main lines of argument that might well rescue forces from phlogiston's fate: (a) in many cases we can argue that although new formulations superseded the forces approach (because those accounts are more practical and elegant for theoretical calculations; here especially Lagrangians and Hamiltonians) the forces formulations are, nonetheless, "exactly equivalent" (Feynman 1965: 53).

And even if "exact equivalence" is not given we might then argue that, (b), forces belong to a level of special science which tracks an important and distinctive level of ontology. That is, even if forces should not be found on the deep level of a grand unified ultimate physics, still they must be recoverable as approximations which are extremely accurate over a wide range of familiar macroscopic events (cf. Feynman 1965: $\left.\int 19-20\right)$ ). The alleged forceless fundamental theory has to explain why and how forces belong to the central phenomenal experiences in the macroscopic world. That is, we ought to be able to identify elements from the ultimate physical theory that correspond to both higher level Newtonian and phenomenologically felt forces.

Clearly, there is some sort of a drawback for (Force) if (b) comes true: what has started as Armstrong's nomological necessity, N, degenerated via (Force) to whatever fundamental feature of the world forces are mere shadows of. Now, if (b) comes true a bold, maybe a little speculative, metaphysical suggestion with which I wish to end the paper could help.

At this point, we might want to merge with (Force) a strategy from above which we did not totally reject, namely (N3.1): say that nomological necessity, $\mathrm{N}$, indeed holds between vast, hyper-complex universals the instantiation of which guaranties the respective effect. This would make allegations (ii) and (iv) less pressing as $\mathrm{N}$ could still be attributed to whatever fundamental laws physics discovers. Now add that $\mathrm{N}$ makes itself known for finite creatures like us as localised component forces. These local forces can be seen as the many causal influences that, altogether, are the instantiation of global $\mathrm{N}$. Causal influence of forces is local (Force) while causation happens indeed via (N3.1) ${ }^{\text {s }}$ totality causes and their effects.

While these thoughts can only serve as a beginning of a follow up paper, the force laws seem in any case to be a good starting point for future considerations about the (alleged) necessity of laws of nature.

\title{
BIBLIOGRAPHY
}

\author{
Author, M. 2007a. \\ Author, M. 2007b. \\ Author, M. 2010a. \\ Author, M. 2010b. \\ Armstrong, D. M. 1978. A Theory of Universals, Vol II. Universals and Scientific Realism. \\ Cambridge: Cambridge University Press. \\ Armstrong, D. M. 1983. What is a Law of Nature. Cambridge: Cambridge University Press. \\ Armstrong, D. M. 1997. A World of States of Affairs. Cambridge: Cambridge University Press. \\ Armstrong, D. M. 2004. Truth and Truthmakers. Cambridge: Cambridge University Press.
}

\footnotetext{
31 In addition to those mentioned in footnote 30, I here add (Wilson 2007) who defends forces (but not component forces) against reduction.
} 
Baxter, D. 2001. 'Instantiation as Partial Identity', Australasian Journal of Philosophy 79: 449-464.

Bigelow, J., Ellis, B., and Pargetter, R. 1988. 'Forces', Philosophy of Science 55: 614-630.

Bigelow, J., and Pargetter, R. 1990. 'Metaphysics of Causation', Erkenntnis 33: 89-119.

Bird, Alexander 2007. Nature's Metaphysics. Dispositions, Laws, and Properties. Oxford: Clarendon Press.

Cartwright, N. 1983. How the Laws of Physics Lie. Oxford: Oxford University Press.

Cartwright, N. 1992. 'Aristotelian Natures and the Modern Experimental Method', in J. Earman (ed.), Inference, Explanation, and other Frustrations: Essays in the Philosophy of Science. Berkeley: University of California Press.

Corry, R. 2006. 'Causal Realism and the Laws of Nature'. Philosophy of Science 73: 261-276.

Creary, L. 1981. 'Causal Explanation and the Reality of Natural Component Forces'. Pacific Philosophical Quarterly 62: 148-157.

Ellis, Brian 2001. Scientific Essentialism. Cambridge: Cambridge University Press.

Feynman, R. 1965. The Character of Physical Law. Cambridge, MA: MIT Press.

Hüttemann, A. 1998. 'Laws and Dispositions', Philosophy of Science 65: 121-135.

Kistler, M. 2003. 'Laws of Nature, Exceptions and Tropes', Philosophia Scientiae 7: 189-219.

Lipton, P. 1999. 'All Else Being Equal', Philosophy 74: 155-168.

Lewis, D. 1986. 'Causation', here quoted from his Philosophical Papers II, Oxford: Oxford University Press: 159-213.

Massin, O. 2009. 'The Metaphysics of Forces'. dialectica 63.4: 555-589.

Molnar, G. 2003. Powers. Oxford: Oxford University Press.

Mumford, S. and Lill Anjum, R. (2011) 'Spoils to the Vector. How to model causes if you are a realist about powers'. The Monist 94 (forthcoming).

Russell, B. 1912. 'On the Notion of Cause'. Presidential Address to the Aristotelian Society Nov 1912. Here quoted from Russell on Metaphysics. Selection from the Writings of Bertrand Russell. Stephen Mumford (ed), London/New York: Routledge 2003.

Tooley, M. 1977 'The Nature of Laws', Canadian Journal of Philosophy 7: 667-698.

van Fraassen, B. 1989. Laws and Symmetry. Oxford: Oxford Clarendon Press.

Wilson, J. 2007. 'Newtonian Forces', The British Journal for Philosophy of Science 58: 173-205.

Wilson, A. 2009. 'Disposition-manifestations and Reference-frames', dialectica 63.4: 591-601. 